\title{
Graphene mode-locked Cr:LiSAF laser at 850 nm
}

\author{
Ferda Canbaz, ${ }^{1}$ Nurbek Kakenov, ${ }^{2}$ Coskun Kocabas, ${ }^{2}$ Umit Demirbas, ${ }^{3}$ and Alphan Sennaroglu ${ }^{1, *}$ \\ ${ }^{1}$ Departments of Physics and Electrical-Electronics Engineering, Laser Research Laboratory, Koç University, Istanbul 34450, Turkey \\ ${ }^{2}$ Department of Physics, Bilkent University, Ankara 06800, Turkey \\ ${ }^{3}$ Department of Electrical and Electronics Engineering, Antalya International University, Antalya 07190, Turkey \\ *Corresponding author: asennar@ku.edu.tr
}

Received 16 June 2015; revised 25 July 2015; accepted 10 August 2015; posted 11 August 2015 (Doc. ID 243055); published 28 August 2015

We report, for the first time to our knowledge, a mode-locked femtosecond Cr:LiSAF laser initiated with a high-quality monolayer graphene saturable absorber (GSA), synthesized by chemical-vapor deposition. The tight-focusing resonator architecture made it possible to operate the Cr:LiSAF laser with only two $135 \mathrm{~mW}, 660 \mathrm{~nm}$ low-cost single-mode diode lasers. At a pump power of $270 \mathrm{~mW}$, the laser produced nearly transform-limited $68 \mathrm{fs}$ pulses with an average power of $11.5 \mathrm{~mW}$ at $850 \mathrm{~nm}$. The repetition rate was around $132 \mathrm{MHz}$, corresponding to a pulse energy and peak power of $86 \mathrm{pJ}$ and $1.26 \mathrm{~kW}$, respectively. Once mode locking was initiated with the GSA, stable, uninterrupted femtosecond pulse generation could be sustained for hours. The saturation fluence and the modulation depth of the GSA were further determined to be $28 \mu \mathrm{J} / \mathrm{cm}^{2}$ and $\mathbf{0 . 6 2 \%}$, respectively. () 2015 Optical Society of America

OCIS codes: (140.3580) Lasers, solid-state; (140.4050) Mode-locked lasers; (140.5680) Rare earth and transition metal solid-state lasers; (140.7090) Ultrafast lasers; (140.3600) Lasers, tunable; (140.3480) Lasers, diode-pumped.

http://dx.doi.org/10.1364/OL.40.004110

The Cr:LiSAF gain medium, an important member of the class of Cr:colquiriite lasers, has emerged as a versatile solid-state gain medium with broad, tunable emission in the $780-1110 \mathrm{~nm}$ wavelength range [1]. Its high emission cross section-fluorescence lifetime product $\left(322 \mu \mathrm{s} \times 10^{-20} \mathrm{~cm}^{2}\right)$ and high intrinsic slope efficiency $(>50 \%)$ have made it possible to obtain efficient, low-threshold lasing with the use of widely available low-power, single-mode red pump diodes [1]. The broad emission bandwidth of Cr:LiSAF has further enabled the generation of femtosecond pulses as short as $10 \mathrm{fs}$ [2] and mode-locked tuning over the 800-920 nm wavelength range [3].

One disadvantage of the Cr:colquiriites in general and of Cr:LiSAF in particular is the low value of the nonlinear refractive index $\left(0.8 \times 10^{-16} \mathrm{~cm}^{2} / \mathrm{W}\right.$, four times lower than that for Ti:sapphire [4]), which makes it quite challenging to obtain a sufficient modulation depth via Kerr lens mode (KLM) locking alone. Hence, saturable absorbers and alternative modulation schemes have been proposed recently to improve the long-term stability of the laser during mode-locked operation. For example, semiconductor saturable absorber mirrors (SESAMs) [5] and single-walled carbon nanotubes (SWCNTs) [6] were successfully employed in previous studies to generate mode-locked pulses from Cr:LiSAF lasers with improved long-term stability. In the case of SESAMs, one major drawback is the complexity of the fabrication process. Furthermore, the resonant nature of the quantum well limits the operational bandwidth of the mode-locked laser, making it difficult to obtain pulses with a duration shorter than $25 \mathrm{fs}$ in Cr:LiSAF [7]. In the case of SWCNTs, although the fabrication process is easier and more cost-effective in comparison with that for SESAMs, bandwidth limitations may still arise, depending on the radius distribution of the carbon nanotubes. Furthermore, as the operation wavelength decreases toward the visible, the transmission of the carbon nanotubes increases because of the onset of metallic effects, leading to higher nonsaturable insertion losses [8]. In more recent studies [3,9], our groups further demonstrated the use of a gain-matched output coupler (GMOC) to balance the unwanted gain filtering effects during weak KLM action, resulting in robust and efficient mode-locked operation of Cr:LiSAF lasers. The GMOC technology works effectively in mode-locked lasers with weak Kerr nonlinearities, but the mirrors require a complex design procedure that is specifically optimized based on the spectral emission characteristics of the particular gain medium.

An attractive alternative involves the use of graphene saturable absorbers (GSAs) due to several favorable characteristics. First, there is no practical bandwidth limitation during modelocked operation, since the GSA provides nearly constant single-pass absorption of about $2.3 \%$ over a wide spectral range $(700 \mathrm{~nm}-25 \mu \mathrm{m})$ due to its zero bandgap energy [10]. Second, the fabrication of monolayer graphene and its transfer onto dielectric substrates involves a series of straightforward and cost-effective steps. However, one remaining challenge, especially in the case of Cr:colquiriite lasers pumped with lowpower single-mode diodes, is the low level of optical gain, which may not be sufficient to overcome the small-signal insertion loss ( 4.6\%) of the GSA. Hence, the generation of solitary pulses with a GSA mode-locked Cr:LiSAF laser requires a resonator with a tight focusing geometry as well as low-loss, dispersion-optimized optics. To date, the GSA has been used to generate mode-locked pulses from numerous bulk solid-state 


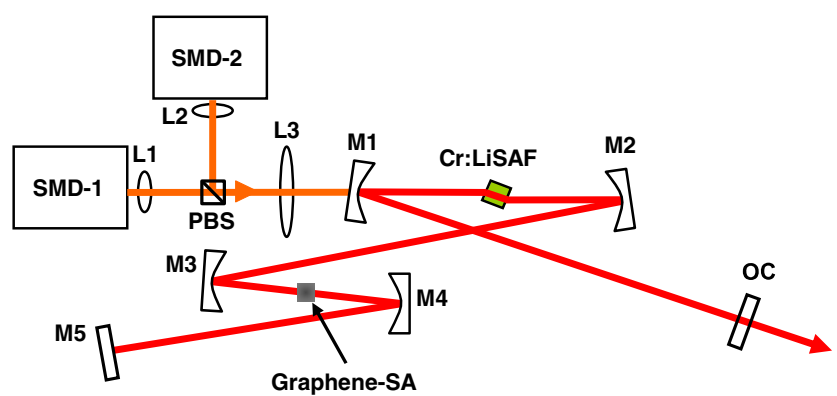

Fig. 1. Schematic of the single-mode diode-pumped Cr:LiSAF laser mode locked with a monolayer graphene saturable absorber.

lasers including Ti:sapphire [11], Cr:YAG [12], Cr:forsterite [13], and Cr:ZnSe [14].

In this study we report, for the first time to our knowledge, the mode-locked operation of a Cr:LiSAF laser, initiated with a high-quality monolayer GSA. The tight-focusing geometry of the resonator enabled the operation of the Cr:LiSAF laser with only two $135 \mathrm{~mW}, 660 \mathrm{~nm}$ low-cost single-mode diode lasers, even after the insertion of the GSA. Above $135 \mathrm{~mW}$ of input pump power, continuous-wave (cw) mode locking could be initiated. At a pump power of $270 \mathrm{~mW}$, the laser produced $68 \mathrm{fs}$ pulses with an average power of $11.5 \mathrm{~mW}$ at the center wavelength of $850 \mathrm{~nm}$. The repetition rate was around $132 \mathrm{MHz}$, corresponding to a pulse energy and peak power of $86 \mathrm{pJ}$ and $1.26 \mathrm{~kW}$, respectively. Once mode locking was initiated with the GSA, stable, uninterrupted femtosecond pulse generation could be sustained for hours.

Figure 1 shows a schematic of the GSA mode-locked femtosecond Cr:LiSAF laser cavity. The output beam of each single-mode pump diode (SMD-1 and SMD-2 in Fig. 1) was collimated with an aspheric lens (L1 and L2, focal length $=4.5 \mathrm{~mm}$ ). The output polarizations of SMD-1 and SMD-2 were adjusted to be orthogonal so that the two pump beams could be polarization coupled with a polarizing beam splitter (PBS). The combined pump beam, which then entered the resonator through the plano-concave input pump mirror (M1), was focused with an input lens (L3, focal length $=60 \mathrm{~mm}$ ) inside the gain crystal. The Brewster-cut, 7-mm-long Cr:LiSAF gain crystal had a chromium concentration of $1.5 \%$ and absorbed $98 \%$ of the $660 \mathrm{~nm}$ pump. The astigmatically compensated x-cavity contained two curved high reflectors (M1 and M2, radius of curvature $=75 \mathrm{~mm}$ ), the gain crystal positioned at Brewster incidence between $\mathrm{M} 1$ and $\mathrm{M} 2$, a flat-end high reflector (M5), and a $0.5 \%$ transmitting output coupler (OC). Furthermore, the GSA was positioned at Brewster incidence in between two additional concave high reflectors (M3 and M4, radius of curvature $=75 \mathrm{~mm}$ ). The high reflector and output coupler arm lengths were 62 and $41 \mathrm{~cm}$, respectively, giving an estimated beam waist of $30 \mu \mathrm{m}$ on the GSA sample. The total cavity length was $1.12 \mathrm{~m}$, corresponding to a repetition rate of $132 \mathrm{MHz}$.

The graphene sample was synthesized by chemical vapor deposition on ultrasmooth copper foils (B1-SBS, Mitsui mining and smelting company, Ltd.). The copper foils were heated to $1035^{\circ} \mathrm{C}$ under $\mathrm{H}_{2}$ flow to reduce the oxide layer of the copper foils. During the growth, the partial pressure of $\mathrm{CH}_{4}$ and $\mathrm{H}_{2}$ gases were 1.5 and 3 Torr, with corresponding flow rates of 40 and $80 \mathrm{sccm}$, respectively. After $30 \mathrm{~min}$, the growth was terminated by stopping the flow of methane, and then the

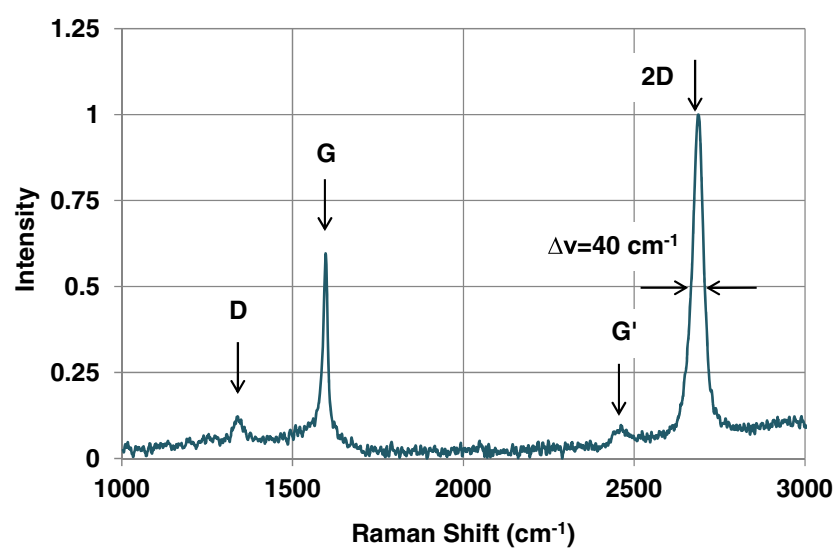

Fig. 2. Measured Raman spectrum of the graphene on Infrasil. The pump wavelength was $532 \mathrm{~nm}$.

samples were cooled down to room temperature under hydrogen flow. In order to transfer the graphene layer onto an Infrasil window, we coated the samples with thick photoresist film (Shipley 1813) and etched the copper in $\mathrm{FeCl}_{3}$ solution. The photoresist film was then placed on the Infrasil window and heated to $120^{\circ} \mathrm{C}$ to initiate uniform coating on the surface. Cleaning the photoresist layer with acetone yielded large-area, high-quality graphene film on the Infrasil window.

In the spectroscopic characterization of the graphene sample, the Raman spectrum was first measured with a Renishaw inVia Raman microscope. The pump wavelength was $532 \mathrm{~nm}$. As has been shown in previous studies, relative intensities and widths of the Raman spectrum give information about the number of layers as well as the quality of the graphene sheet [15]. For the graphene-on-Infrasil sample that was synthesized in our experiments, all of the four characteristic bands (designated as D, G, G', and 2D) could be clearly identified, as can be seen in Fig. 2. In addition, the intensity ratio of the $D$ and $G$ bands $\left(\mathrm{I}_{\mathrm{D}} / \mathrm{I}_{\mathrm{G}}\right)$ and the full width at half maximum (FWHM, $\Delta \nu$ ) of the $2 \mathrm{D}$ band were measured to be 0.18 and $40 \mathrm{~cm}^{-1}$, respectively, suggesting that the GSA used in our experiments was monolayer and nearly free of structural defects. The measured values of $\mathrm{I}_{\mathrm{D}} / \mathrm{I}_{\mathrm{G}}$ and $\Delta \nu$ are in good agreement with previously reported values [16].

Ultrafast dynamics and absorption saturation behavior of the GSA were further investigated with a commercial timeresolved pump-probe spectrometer (HELIOS, Ultrafast systems). Two-color pump-probe measurement was performed by using two tunable optical parametric oscillators (TOPAS; Newport Spectra Physics), each seeded with a commercial Ti:sapphire chirped-pulse amplifier (Spitfire ACE, Newport Spectra Physics), to provide the pump $(850 \mathrm{~nm})$ and probe $(950 \mathrm{~nm})$ beams. Figure 3(a) shows the variation of the fractional transmission $(\Delta T / T)$ of the probe as a function of delay. The fast and slow decay times of the GSA were determined to be $128 \mathrm{fs}$ and $1.630 \mathrm{ps}$, respectively, in good agreement with previously reported values in the literature [17].

At the delay that gives the maximum fractional transmission change, the probe transmission was further measured as a function of the pump fluence [Fig. 3(b)]. From the analysis of the saturation data, based on a two-level rate equation model similar to what is described in Ref. [18], the best-fit values of the saturation fluence and modulation depth were determined to 

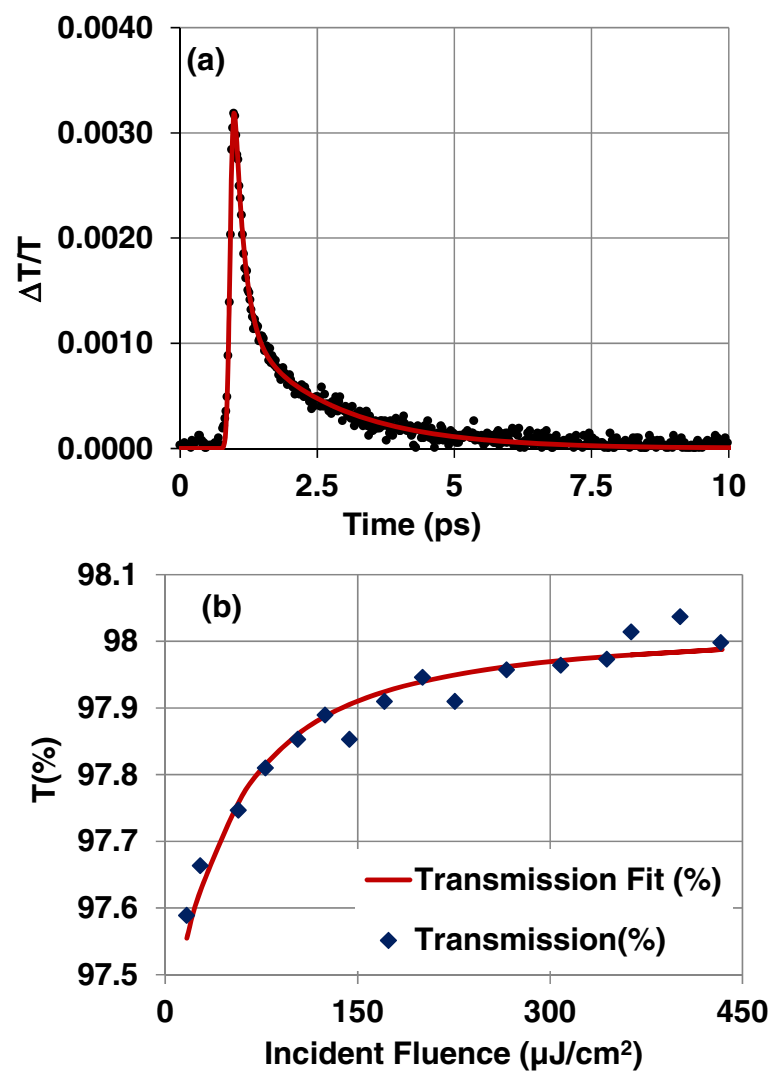

Fig. 3. (a) Measured variation of the fractional transmission $(\Delta T / T)$ of the probe $(950 \mathrm{~nm})$ as a function of delay; (b) variation of the probe transmission as a function of the incident fluence at the wavelength of $850 \mathrm{~nm}$ for the GSA.

be $28 \mu \mathrm{J} / \mathrm{cm}^{2}$ and $0.62 \%$, respectively. The small-signal transmission of the sample was determined to be $97.5 \%$ from absorbance measurements. The reason for the deviation of the modulation depth from the theoretical value of $2.3 \%$ requires further investigation and may be due to crystal imperfections that form during synthesis.

Figure 4(a) shows the measured threshold pump power of the $\mathrm{cw}$ Cr:LiSAF laser at different output coupling levels. By measuring the variation of the threshold pump power, the small-signal loss of the crystal was estimated to be $0.55 \%$. Figure 4 (b) further shows the variation of the cw output power as a function of the pump power (free-running resonator) for different output coupling levels. With a total pump power of $270 \mathrm{~mW}$, output power as high as $101 \mathrm{~mW}$ was obtained by using the $1 \%$ transmitting output coupler. Since the inclusion of the GSA provides additional losses of about $2.5 \%$ per transit, mode-locking experiments were conducted by using the $0.5 \%$ transmitting output coupler to provide higher intracavity intensity. Once the GSA was inserted into the cavity as shown in Fig. 1, cw mode-locked operation could be readily obtained by translating the output coupler. Inclusion of the GSA reduced the maximum cw output power of the laser from 86 to $10 \mathrm{~mW}$, as shown in Fig. 4(c). By comparing the threshold pump powers of the laser with and without the GSA, the round-trip insertion loss of the GSA was estimated to be $4.9 \%$, in good agreement with twice the measured small-signal absorption of $5 \%$.
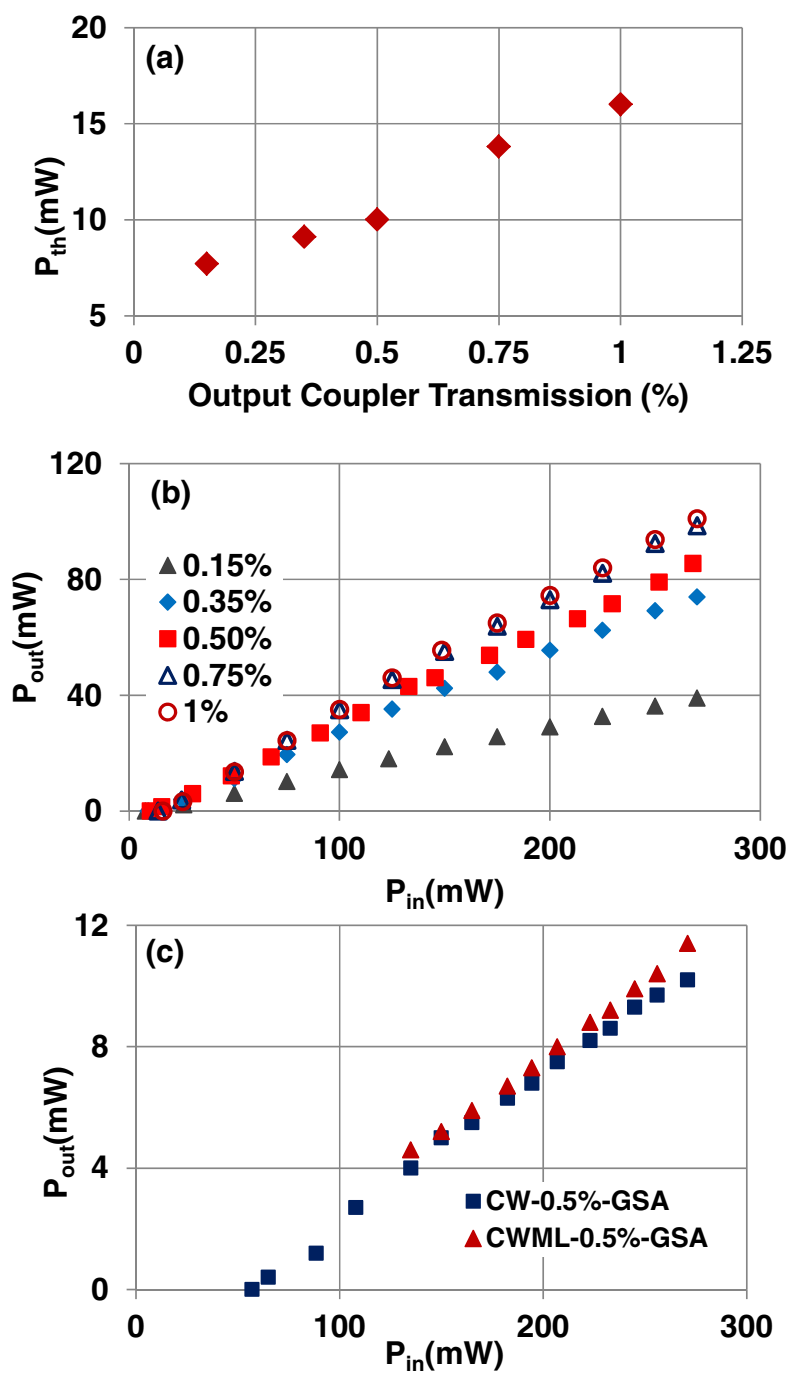

Fig. 4. (a) Measured threshold pump power as a function of the output coupler transmission for the cw cavity; (b) measured variation of the free-running laser output power as a function of the incident pump power at different output coupling levels; (c) cw and cw modelocked (CWML) power performance of the Cr:LiSAF laser containing the GSA and the $0.5 \%$ output coupler.

Figures 5(a)-5(c) show the measured optical spectrum, interferometric autocorrelation, and radio frequency (RF) spectrum of the femtosecond pulses generated with the GSA mode-locked Cr:LiSAF laser. At the center wavelength of $850 \mathrm{~nm}$, the pulse duration and spectral bandwidth measurements yielded $68 \mathrm{fs}$ (assuming a sech ${ }^{2}$ intensity profile) and $12 \mathrm{~nm}$, respectively, with a corresponding time-bandwidth product of 0.338 , indicating that the pulses were nearly transform limited. Furthermore, in the RF spectrum measurements shown in Fig. 5(c), the sideband noise level was determined to be $70 \mathrm{~dB}$ below the fundamental tone at $132 \mathrm{MHz}$ (resolution bandwidth $=1 \mathrm{kHz}$ ).

The mode-locking data shown in Figs. 5(a)-5(c) were taken at an input power of $270 \mathrm{~mW}$ with a $0.5 \%$ output coupler. The maximum mode-locked output power was $11.5 \mathrm{~mW}$. As can be seen in Fig. 4(c), somewhat higher output powers were obtained during mode-locked operation in comparison with the $\mathrm{cw}$ case, since the saturated absorption of the GSA at 

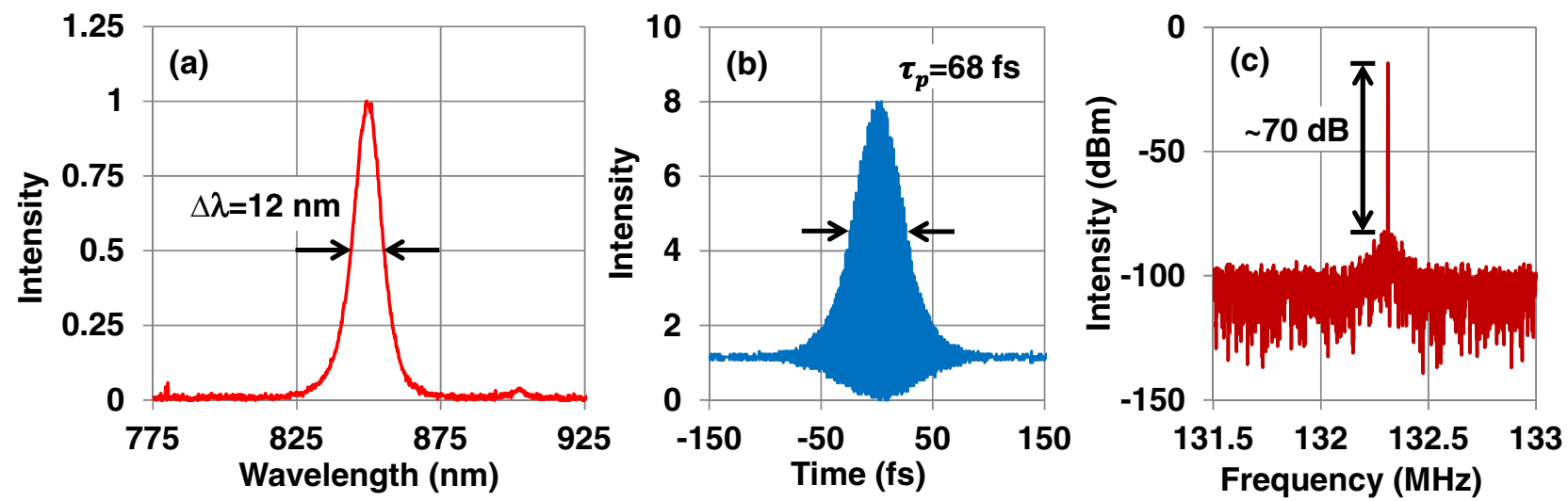

Fig. 5. Measured (a) optical spectrum; (b) interferometric autocorrelation trace; and (c) radio frequency spectrum of the $68 \mathrm{fs}$ pulses generated with the GSA mode-locked Cr:LiSAF laser near $850 \mathrm{~nm}$ at a pulse repetition rate of $132 \mathrm{MHz}$.

higher intracavity peak powers leads to lower overall resonator loss during mode-locked operation. The corresponding maximum fluence on the GSA was determined to be $606 \mu \mathrm{J} / \mathrm{cm}^{2}$, suggesting that based on our saturation measurements, the GSA was fully saturated. Pure and stable mode-locking operation could be initiated at any pump power level above $130 \mathrm{~mW}$ (corresponding output power $=4 \mathrm{~mW}$ ). As the input power was decreased, initiation of mode locking became more difficult. Below $130 \mathrm{~mW}$ of pump power, pulsed operation could not be initiated. In this regime, although a $Q$-switching tendency was observed by translating the output coupler mirror, stable $Q$ switching could not be obtained.

To obtain solitary pulses, dispersion management was employed by using commercial and custom-made dispersioncontrol optics [M1 and M2, each with $-80 \pm 10 \mathrm{fs}^{2}$ of group delay dispersion (GDD) per bounce; M5, with $-40 \pm 10 \mathrm{fs}^{2}$ of GDD per bounce]. The material dispersion contributions of the gain crystal and Infrasil substrate were +24 and $40 \mathrm{fs}^{2}$ per $\mathrm{mm}$, respectively. Because the net GDD of the cavity was close to zero, it was not possible to determine the overall dispersion from the nominal GDD values given above, since the actual GDD values of the mirror coatings are uncertain due to the dependence on the angle of incidence. Instead, based on the soliton area theorem [19] and the nonlinear refractive index of Cr:LiSAF $\left(0.8 \times 10^{-20} \mathrm{~m}^{2} / \mathrm{W}\right)$ [4], the net round-trip cavity GDD was estimated to be $-75 \mathrm{fs}^{2}$.

In conclusion, we reported the first demonstration of a mode-locked femtosecond Cr:LiSAF laser, initiated with a high-quality monolayer GSA. In comparison with the SWCNT mode-locked Cr:LiSAF lasers described in Ref. [6], the operation of the graphene mode-locked Cr:LiSAF laser described here was more stable, possibly due to the larger modulation depth of the GSA. Furthermore, since graphene poses no practical bandwidth limitation, unlike SWCNTs or SESAMs, we foresee the generation of few-cycle pulses with this source by using dispersion-optimized resonator optics.

Funding. TUBITAK (112T967, 114F185).

Acknowledgment. The authors thank Hakan Urey for providing some of the measurement equipment, and Natali
Çizmeciyan and Barış Yağcı (KUYTAM, Koç University Surface Science and Technology Center) for help during spectroscopic measurements.

\section{REFERENCES}

1. U. Demirbas, D. Li, J. R. Birge, A. Sennaroglu, G. S. Petrich, L. A. Kolodziejski, F. X. Kaertner, and J. G. Fujimoto, Opt. Express 17, 14374 (2009).

2. S. Uemura and K. Torizuka, Jpn. J. Appl. Phys. 39, 3472 (2000).

3. C. Cihan, E. Beyatli, F. Canbaz, L. J. Chen, B. Sumpf, G. Erbert, A. Leitenstorfer, F. X. Kartner, A. Sennaroglu, and U. Demirbas, IEEE J. Sel. Top. Quantum Electron. 21, 94 (2015).

4. E. Sorokin, Few-Cycle Laser Pulse Generation and Its Applications, F. X. Kartner, ed. (Springer-Verlag, 2004), pp. 3-71.

5. D. Kopf, K. J. Weingarten, G. Zhang, M. Moser, M. A. Emanuel, R. J. Beach, J. A. Skidmore, and U. Keller, Appl. Phys. B 65, 235 (1997).

6. A. Agnesi, F. Pirzio, E. Ugolotti, S. Y. Choi, D. I. Yeom, and F. Rotermund, Opt. Commun. 285, 742 (2012).

7. U. Demirbas, G. S. Petrich, D. Li, A. Sennaroglu, L. A. Kolodziejski, F. X. Kärtner, and J. G. Fujimoto, J. Opt. Soc. Am. B 28, 986 (2011).

8. H. Kataura, Y. Kumazawa, Y. Maniwa, I. Umezu, S. Suzuki, Y. Ohtsuka, and Y. Achiba, Synth. Met. 103, 2555 (1999).

9. F. Canbaz, E. Beyatli, L. J. Chen, A. Sennaroglu, F. X. Kartner, and U. Demirbas, Opt. Lett. 39, 327 (2014).

10. A. B. Kuzmenko, E. van Heumen, F. Carbone, and D. van der Marel, Phys. Rev. Lett. 100, 117401 (2008).

11. I. H. Baek, H. W. Lee, S. Bae, B. H. Hong, Y. H. Ahn, D.-I. Yeom, and F. Rotermund, Appl. Phys. Express 5, 032701 (2012).

12. S. D. D. Cafiso, E. Ugolotti, A. Schmidt, V. Petrov, U. Griebner, A. Agnesi, W. B. Cho, B. H. Jung, F. Rotermund, S. Bae, B. H. Hong, G. Reali, and F. Pirzio, Opt. Lett. 38, 1745 (2013).

13. W. B. Cho, J. W. Kim, H. W. Lee, S. Bae, B. H. Hong, S. Y. Choi, I. H. Baek, K. Kim, D.-I. Yeom, and F. Rotermund, Opt. Lett. 36, 4089 (2011).

14. M. N. Cizmeciyan, J. W. Kim, S. Bae, B. H. Hong, F. Rotermund, and A. Sennaroglu, Opt. Lett. 38, 341 (2013).

15. A. C. Ferrari, J. C. Meyer, V. Scardaci, C. Casiraghi, M. Lazzeri, F. Mauri, S. Piscanec, D. Jiang, K. S. Novoselov, S. Roth, and A. K. Geim, Phys. Rev. Lett. 97, 187401 (2006).

16. A. Reina, X. Jia, J. Ho, D. Nezich, H. Son, V. Bulovic, M. S. Dresselhaus, and J. Kong, Nano Lett. 9, 30 (2009).

17. J. M. Dawlaty, S. Shivaraman, M. Chandrashekhar, F. Rana, and M. G. Spencer, Appl. Phys. Lett. 92, 042116 (2008).

18. A. Sennaroglu, U. Demirbas, S. Ozharar, and F. Yaman, J. Opt. Soc. Am. B 23, 241 (2006).

19. H. A. Haus, IEEE J. Sel. Top. Quantum Electron. 6, 1173 (2000). 